\title{
A novel acoustic imaging system based on digital MEMS sensors
}

\author{
Linsong $\mathrm{CHEN}^{1, \mathrm{a}}$, Yueyun $\mathrm{CAO}^{2, \mathrm{~b}}$ and Wenyong $\mathrm{GUO}^{1}$ \\ ${ }^{1}$ Power Eng. College, Naval Univ. of Engineering, Wuhan 430033, China; \\ ${ }^{2}$ Electronics Eng. College, Naval Univ. of Engineering, Wuhan 430033, China. \\ alinsirchen@163.com, ${ }^{\mathrm{b}} 653143156 @ q q . c o m$
}

Keywords: acoustic imaging; MEMS microphone; FPGA; DSP; beamforming.

\begin{abstract}
Developed an acoustic imaging system applied in acoustic test. The acoustic array is made up of 260 MEMS microphones and control by a FPGA, which also in charge of transferring data by optical fiber. The mainframe is control by a FPGA. A DSP is in charge of processing the data from acoustic array. Two embedded system work for saving data and displaying acoustic image. Test results verified that the system can satisfy the need of acoustic imaging for machinery noise and gas-leakage noise in normal workshop. This system has a wide application.
\end{abstract}

\section{Introduction}

Acoustic array in air is a powerful tool to analysis the radiation of complex sound source. With a single measurement in a short time, one can get the acoustic image, which shows the sound distribution condition and frequency characteristics[1][2]. It can be used for sound source location, mechanical noise fault diagnosis and environmental noise monitoring.

To improve the quality of acoustic image, researchers are hard at increasing the number of microphone sensors on an efficient measurement surface.

Traditional array is made of electret capacitor microphone, which is in big size with extremely precise. But the array made of this kind of microphone is complex with a huge data acquisition system[3]. The number of the microphones is usually limits to 30 . The acoustic image is not satisfactory for most condition. Though move array is designed to increase the numbers of measurement point[4][5][6], it is used only for a steady-state sound field.

In this paper, an acoustic array based on MEMS microphone is design. The numbers of the sensors of the array is increase to 260 . The data acquisition system is integrated tightly together with the array with a high processing speed. This imaging system can get continuous dynamic images with high-resolution of any stationary or unstationary sound field.

\section{System design}

As show in figure 1, there is 260 MEMS microphones in the front-end. A FPGA is in charge of the data acquisition work and fiber transmission. A camera is set in the center of the array to get the actual photo of the test target. In the back-end, another FPGA is in charge of the system. The fiber transmission modules gets the data from the acoustic array and sent to FPGA by UART (Universal Asynchronous Receiver/transmitter). Array signal processing work such as FFT are finished in a piece of DSP. Instants data storage is in charged by a piece of PC104 modules. Another PC104 modules is worked as display and control system, which can transfer the data with DSP and data storage modules by Ethernet. 


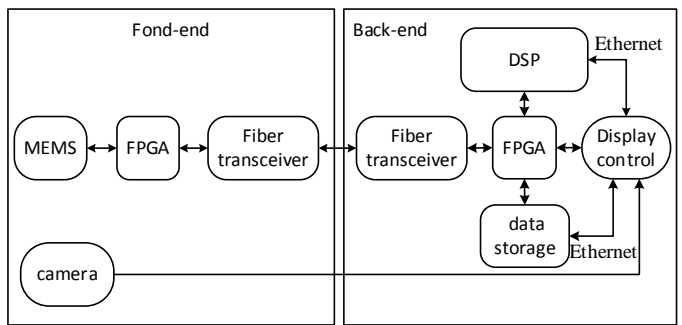

Figure 1. Schematic diagram of acoustic array system based on MEMS.

The block diagram of data acquisition system of font-end is show in Figure 2. Each sensor sent a standard 24-bit sound data to data buffer. The buffer is used to increase the drive capability. Propose the sound sampling speed is set $50 \mathrm{kHz}$, all the 260 sensors should sent the data at $52 \mathrm{MB} / \mathrm{s}$ (sound data is transferred at 32-bit).

The block diagram of data procession system of back-end is show in figure 3 .

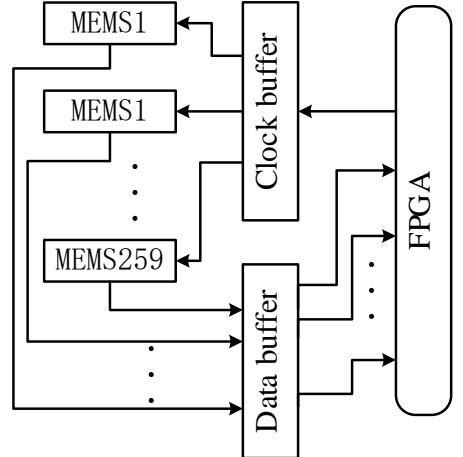

Figure 2. MEMS block control by FPGA

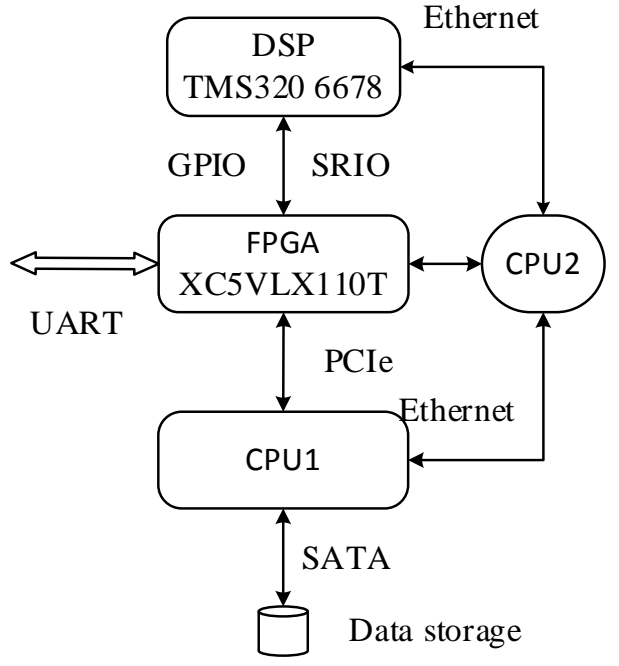

Figure 3. Data procession system of back-end

\section{Array design}

\section{Array design}

Usually, the acoustic sensors on an array use for acoustic holography is uniform distributed, while an array for beamforming is non-uniform distributed. To adapt the array to both acoustic holography and beamfoming using, a tri-nested array is developed. As show in figure 4, the side length of the square array is $0.935 \mathrm{~m}$. Three kinds of grid space $0.085 \mathrm{~m}, 0.0425 \mathrm{~m}$ and $0.0213 \mathrm{~m}$ are provided. As show in figure 4, the microphone distributes closely in center and sparsely on edge. Different grid space correspond to different upper limit of noise frequency. The higher frequency is analysis, the smaller grid space is demanded, and the smaller of the array area is required. 


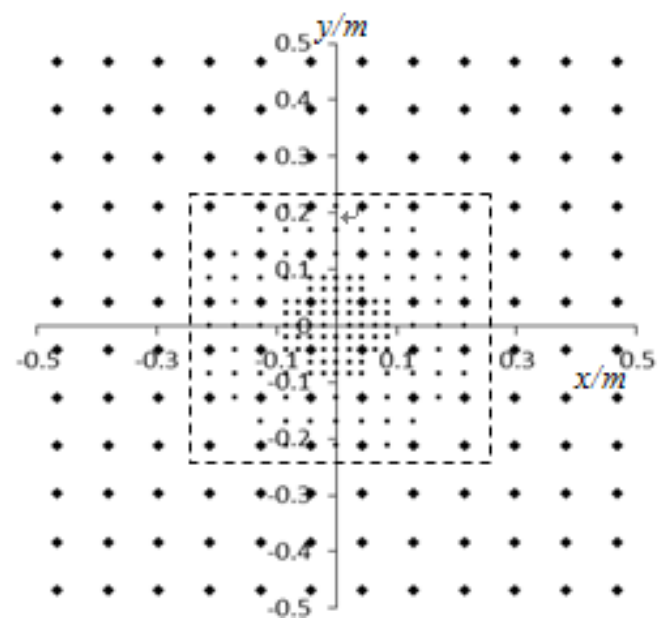

Figure 4. Distribution of microphone in nested array

\section{Performance analysis}

As demonstrated in the theory of array signal procession, quiescent pattern show the array's azimuth resolution ability for noise source. In this system, the pattern represent the imaging performance of the array. Figure 5 shows the quiescent pattern of the array at $3 \mathrm{kHz}$. This array is adapt to a wide noise band from $2 \mathrm{kHz}$ to $15 \mathrm{kHz}$ when working at beamforming mode to get acoustic image.Working at NAH (near-field acoustic holography) mode, this array is adapt to $100 \mathrm{~Hz}$ to $1000 \mathrm{~Hz}$.

\section{Test of microphones}

MEMS microphone has a perfect frequency response on a wide band. As show in user manual of ADMP441, it has a flat frequency response from $60 \mathrm{~Hz}$ to $15 \mathrm{kHz}$. And it is easy to verify by the test system.

To verify the phase coherence of the sensors, a loudspeaker was set $0.3 \mathrm{~m}$ in front of the array in the center. A single analog audio signal at $500 \mathrm{~Hz}$ was produced by the speaker. Figure 6 shows the distribution of the phase of all 260 MEMS microphones. There are almost on a smooth curved surface, the same as the theoretical analysis result.
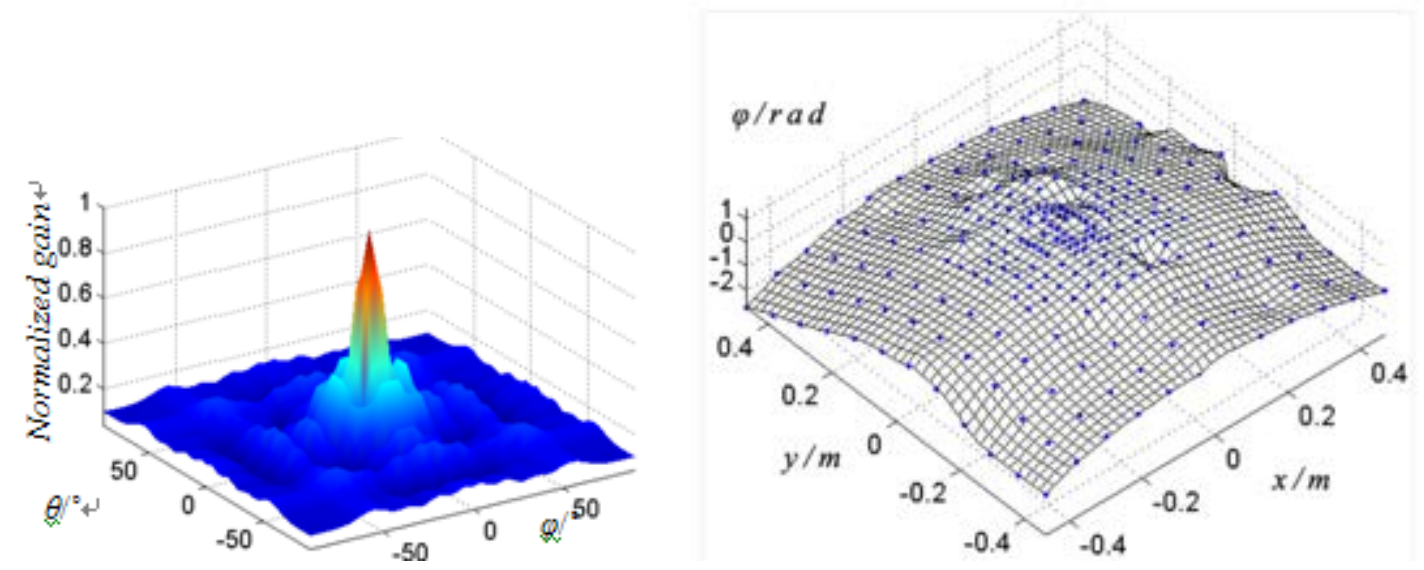

Figure 5. Directivity patterns at $3 \mathrm{kHz}$

Figure 6. Phase equalization test for array cells

When working at NAH (near-field acoustic holography) mode [7], this array is adapt to $100 \mathrm{~Hz}$ to $1000 \mathrm{~Hz}$. 


\section{Experimental test}

A test system is setup to check the imaging quality and anti-interference performance of the image system. Two loudspeakers were set closely in front of the array. Both speaker produced the same coherent noise signal from $100 \mathrm{~Hz}$ to $15 \mathrm{kHz}$.

To get the image of the noise which frequency is bellow $1000 \mathrm{~Hz}$, the array is set 0.1 meter before the loudspeakers. The system work as a NAH imaging system. Figure 6 show the NAH image of the sound source at $200 \mathrm{~Hz}$.

To get the image of the noise which frequency is high than $1000 \mathrm{~Hz}$, the array is set 1 meter or more far from the loudspeakers. The system work as a beamforming[8] imaging system. Figure 7 show the image of the sound source at $5 \mathrm{kHz}$.

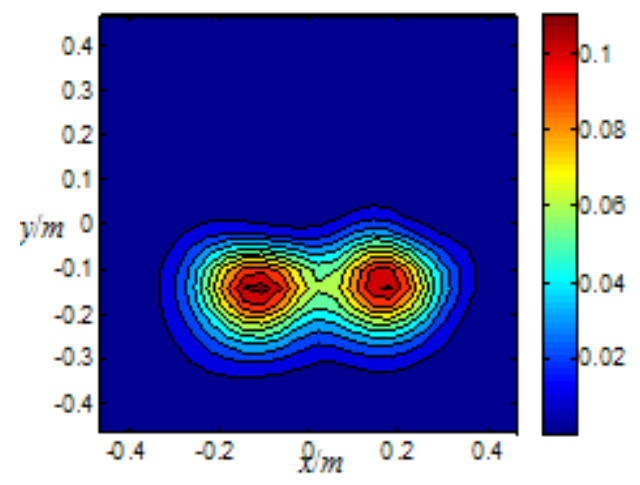

Figure 7. Acoustic holography of noise at $100 \mathrm{~Hz}$

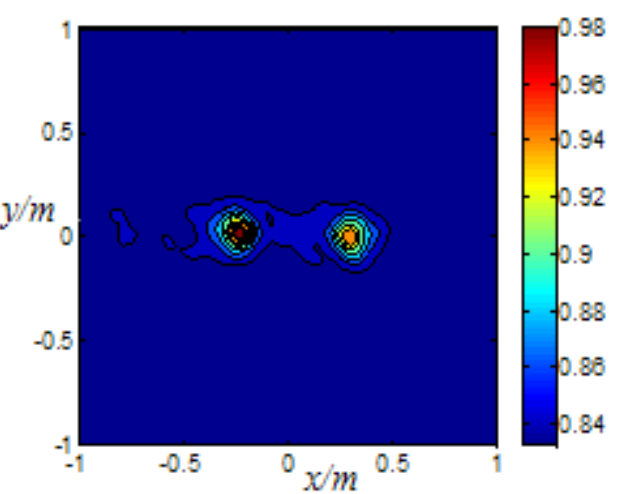

F igure8. Beamforming image of noise at $5 \mathrm{kHz}$

These experiments verified that this imaging system is reliable and effective.

\section{Actual noise imaging experiment}

This system is designed for actually use. Being superior to any former image system which must work in anechoic chamber and measure steady sound source [9], this system can work at an ordinary workroom and measure any steady or unsteady sound source.

An experiment system was setup as show as figure 9. The machinery bench is made up of a rotor, gear case and a oil pump. The distant from the array to the machine is $1.2 \mathrm{~m}$. The noise are unsteady with the strength and frequency varied by time. Two loudspeakers were set to check the imaging ability for multi-source.

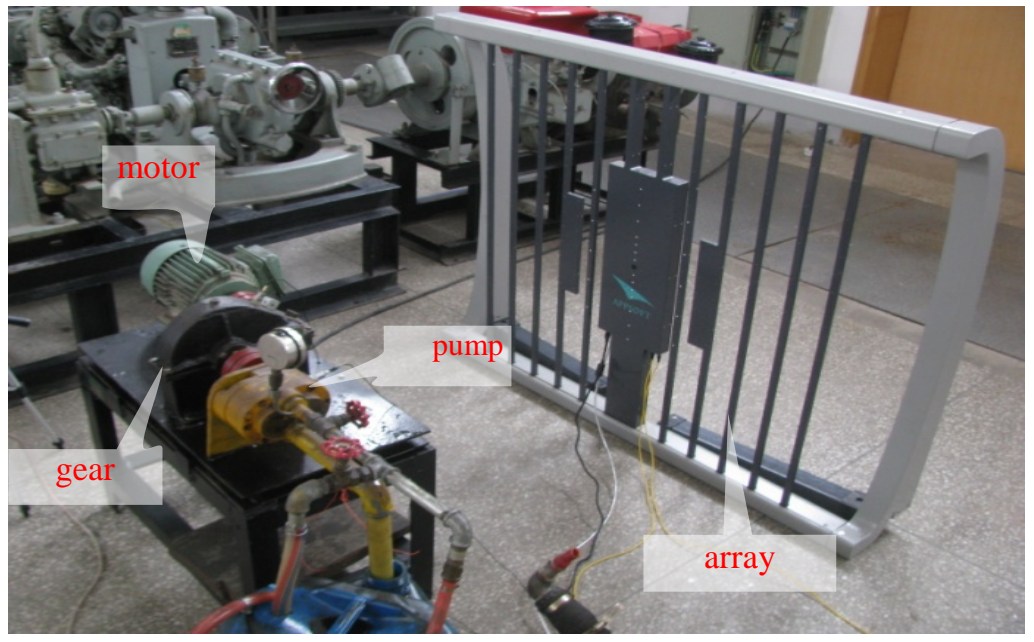

Figure 9. Acoustic imaging system for machinery noise

Figure 10 is one of the beamforming images of the experiment. Three sound sources were located exactly. A dynamic video of the noise can show the variety of the sound sources. 


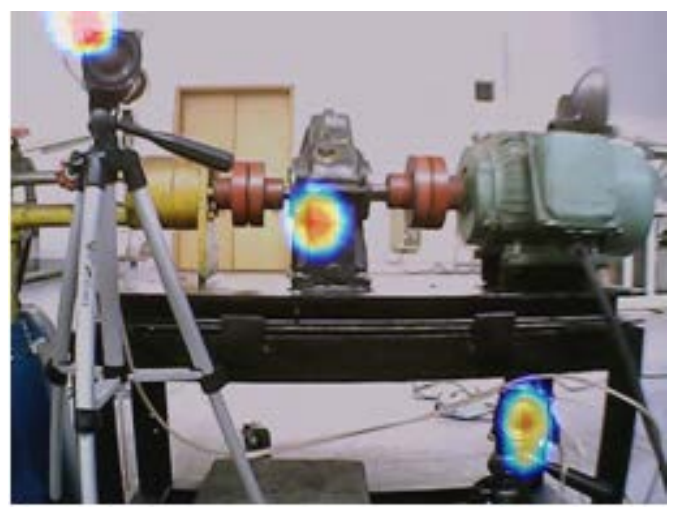

Figure 10. Acoustic imaging of multiplesources

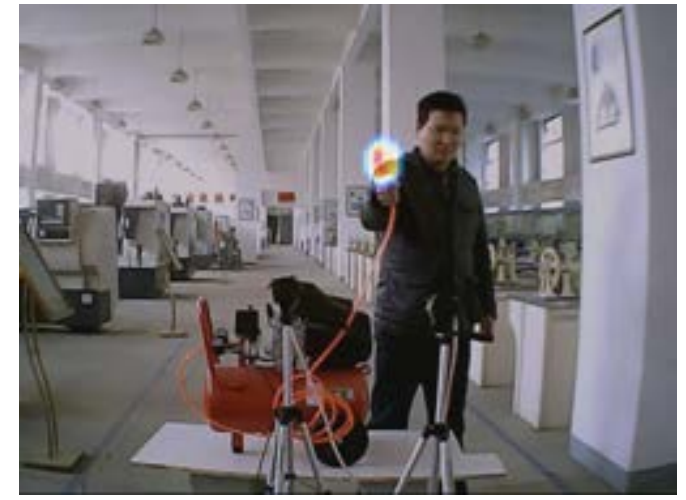

Fig.11 gas-leakage location by acoustic imaging

This system is helpful for locating gas leak source in some case. Because the gas leak noise has a wide spread frequency band than the machinery noise, the gas leak noise can easily be identified and located under the interference of machinery noise.

Figure 11 show the image of gas leak source location experiment. It is a beamforming image form at $8 \mathrm{kHz}$.

\section{Conclusion}

In this paper, an acoustic array imaging system based on MEMS microphones was designed. It has more sensors, more compact appearance, higher resolution image, and higher imaging speed than the acoustic array system which is based on traditional electret microphone[10]. It can be used in ordinary workshop to measure the acoustic images of any stationary or non-stationary sound source such as machine and gas leakage noise.

\section{Reference}

[1] Mingsian R. Bai, Jeong-Guon Ih, Jacob Benesty. Acoustic Array System [M]. Quebec Canada, 2013.

[2] Earl G. Williams and Brian H. Houston. Interior near-field acoustical holography in flight. J. Acoust. Soc. Am. 108 (4), October 2000:1451-1463.

[3] J. Panda, R. Mosher. Use of a Microphone Phased Array to Determine Noise Sources in a Rocket Plume. AIAA control ID: 892750.

[4] Earl G. Williams, Henry D. Dardy. Underwater-nearfield acoustical holography using a robotic controlled scanner. J. Acoust. Soc. Am. 75(1), Spring 1984:s64.

[5] Alfredo Cigada, Massimiliano Lurati, Francesco Ripamonti, Marcello Vanali. Moving microphone arrays to reduce spatial aliasing in the beamforming technique: Theoretical background and numerical investigation[J]. J. Acoust. Soc. Am. 2008, 124(6): 3648-3658.

[6] Hyu-Sang Kwon, Yang-Hann Kima. Moving frame technique for planar acoustic holography [J]. J. Acoust. Soc. Am. 1998, 103 (4): 1734-1341.

[7] Earl G. Williams. Fourier Acoustics[M]. Washington. D.C. 1999.

[8] Philip J. Jackson, Finn Jacobsen. Sound field Planariy characterized by superdirective beamforming[J]. Proceedings of Meetings on Acoustics, Vol.19, 055056.

[9] Mathew Legg and Stuart Bradley. A Combined Microphone and Camera Calibration Technique With Application to Acoustic Imaging[J]. IEEE transactions on image processing, 2013, 22(10): 4023-4039.

[10] Jacob Benesty, Jingdong Chen, and Yiteng Huang. Microphone Array Signal Processing[M]. Springer, Berlin, 2008. 